\title{
Energy-Efficient Cellular Network Operation via Base Station Cooperation
}

\author{
Feng Han, Zoltan Safar, W. Sabrina Lin, Yan Chen and K. J. Ray Liu \\ Department of ECE, University of Maryland, College Park, MD, 20742, USA \\ Email: \{hanf, zsafar, wylin, yan, kjrliu\}@umd.edu
}

\begin{abstract}
The rising cost of energy and increased environmental awareness have sparked a keen interest in the development and deployment of energy-efficient communication technologies. The energy efficiency of cellular networks can be increased significantly by selectively switching off some of the base stations (BSs) during periods of low traffic load. In this paper, we propose a scalable BS switching strategy and use cooperative communications and power control to extend network coverage to the service areas of the switched-off BSs. The outage probability and the achievable power savings of the proposed scheme are analyzed, both analytically and numerically, and a potential of up to $50 \%$ power saving is observed in the numerical results.
\end{abstract}

\section{INTRODUCTION}

The Information and Communications Technology (ICT) industry has experienced an explosive growth during the past few decades, and it continues to grow rapidly. Consuming roughly 900 billion KWh per year, the ICT infrastructure is responsible for about $10 \%$ of the world's electric energy consumption [1]. Within the ICT sector, the mobile telecommunication industry is one of the major contributors to energy consumption. Reports show that nearly half of the total operating expenses for a mobile telecommunication operator is the energy cost [2]. Therefore, energy-efficient cellular network operation is needed more than ever before to reduce both the operational expenses and the carbon footprint of this industry.

In a typical cellular system, base stations (BSs) contribute $60 \%-80 \%$ of the energy consumption of the whole network [3]. Thus, improving the energy-efficiency of the BSs can significantly reduce both the operational cost and carbon footprint. It is worth noting that the energy consumption of a BS is dominated by components that do not depend on the traffic load, such as the high-power tower lights and the cooling mechanism. As an example, a typical BS consumes 800-1500 $\mathrm{W}$, while its power amplifier output is only 40-80 W [4]. This means that a BS consumes more than $90 \%$ of its peak consumption even in periods of idle operation. Therefore, to achieve better energy efficiency, one must take a system-wide approach where entire BSs are turned off in the network in a coordinated manner, and the corresponding traffic load is distributed among the remaining active BSs. If the overall network traffic load is low (e.g. during late evening and night), and the active BSs can use power control and cooperative communications to improve the radio link quality, such an approach has the potential for significant energy savings while being able to provide the desired quality of service (QoS).

Among previous work on energy saving by BS operation management mechanisms, the authors of [5] proposed turning off half of the BSs in a regular pattern and analyzed the call blocking probability and the average number of active calls as functions of the call generation rate. In [3] and [6], the amount of saved energy was characterized for different temporal traffic patterns and switching strategies. A centralized and a decentralized BS switching algorithm in [7] assigned "active" or "sleep" states to BSs and users to "active" BSs based on the transmission rate requirements of the users and the capacity of the BSs. To lower the energy consumption, a heterogeneous cellular architecture was proposed in [8], where additional microcells provided increased capacity during peak hours, but these microcells were turned off during periods with low traffic demand, resulting in a more energy-efficient cellular system. Algorithms for the deployment and operation of such a heterogeneous network were proposed in [9] based on the notion of area spectral efficiency. The authors of [10] considered the scenario where two operators share the same BS during low traffic periods and analyzed the achievable energy savings.

After switching off some BSs, the service areas of the remaining active $\mathrm{BSs}$ increase, reducing the signal to noise ratio (SNR) at the receiver side considerably due to increased distances between the active BSs and the user equipments (UEs). Most previous work did not consider the QoS degradation due to this effect, concentrating on energy saving potential and system capacity issues instead.

The last decade witnessed a lot of progress in cooperative communications [11], and it has been used to efficiently extend network coverage [12] [13]. This technique was applied in [14], where the signal strength outage and service blocking outage of the UEs were characterized, and the achievable energy consumption reduction was studied as the function of the traffic load via computer simulations. A recent survey on the current state of the art of energy-efficient cellular network operation can be found in [4].

In this paper, we propose a scalable BS switching strategy and use cooperative communications and power control to mitigate the SNR degradation due to the switched-off BSs. We analyze the performance of the resulting energy-efficient cellular network and characterize the achievable energy saving. Unlike [14], we consider a number of BS switch-off patterns across a large-scale network, incorporate the effect of multipath fading into our analysis, and derive analytical expressions for the outage probability due to insufficient received SNR for both uplink and downlink transmission scenarios. We focus on UEs with the worst channel conditions instead of the spatial average, which allows us to guarantee system-wide QoS levels. 


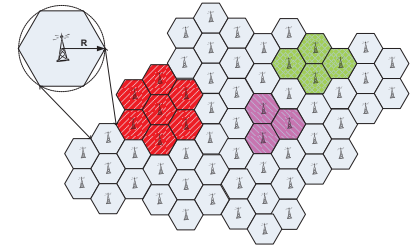

Fig. 1. A cellular network covered by hexagonal cells

\section{SySTEM MODEL AND OPERATION}

\section{A. System Model}

We consider a cellular network covered by hexagonal cells with radius $R$, and each cell is equipped with a multi-antenna BS located in the center of the cell, as shown in Fig. 1. The BSs are inter-connected with backhaul links, through which communications are assumed to be low-cost, reliable and highspeed. Each BS has $M$ antennas, and the UE served by this network has one single antenna. Let $h_{k}^{(m)}$ denote the fading channel coefficient between a UE and the $m$-th antenna of the $k$-th BS. Due to channel reciprocity, these channel coefficients are assumed to be the same in both downlink and uplink directions. They are modeled as circularly symmetric complex Gaussian random variables with zero mean and variance

$$
\sigma_{k}^{2}=\frac{G}{d_{k}^{\alpha}}
$$

where $d_{k}$ is the distance between the $k$-th cell site and the UE, $\alpha$ is the path loss exponent, and $G$ is the normalizing gain factor. Assume that $h_{k}^{(m)}$ 's are uncorrelated for different values of $(k, m)$, and the channel state information is available at the BS side.

\section{B. BS Switching Strategy}

During low-traffic hours, some of the BSs are switched off, resulting in a more energy-efficient network operation, and the corresponding traffic load is distributed among the remaining active BSs. Since the traffic demand during these hours is much lower than during peak hours, the active BSs can easily accommodate the additional traffic load and can schedule all transmissions on orthogonal transmission resources. As a consequence, we assume that this operation will not cause noticeable increase in call blocking probability or higher interference levels. That is, the network becomes coverage-limited rather than capacity-limited when some of the BSs are switched off.

In determining which cell sites to switch off, the optimal solution involves integer programming, which is proven to be NP-hard [15], [16]. Moreover, the previously proposed suboptimal algorithms [7], [9] did not consider the possibility of BS cooperation in their objective functions. Therefore, we propose to use a set of intuitive BS switching patterns that can be used as basic building blocks to cover the entire network. Fig. 2 shows the basic switching pattern and the resulting network topology when one BS is switched off in a basic building block consisting of three BSs. We will refer to this case as switch-off pattern I in the sequel. Similarly, Fig. 3 and Fig. 4 present two more examples, switch-off patterns II and III, corresponding to switching off two BSs out of four and switching off three BSs out of seven BSs, respectively. Defining $\beta$ to be the ratio of the number of switched-off BSs

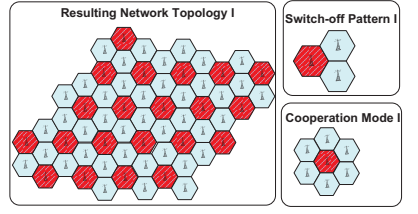

Fig. 2. Switch-off Pattern I: Switching off one out of three BSs

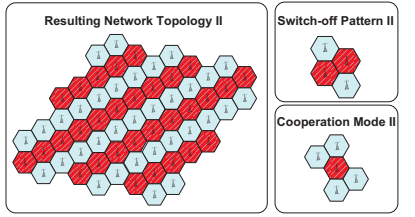

Fig. 3. Switch-off Pattern II: Switching off two out of four BSs

to the total number of BSs for switch-off patterns I, II and III, we obtain $\beta_{I}=1 / 3=33 \%, \beta_{I I}=2 / 4=50 \%$, and $\beta_{I I I}=4 / 7=57 \%$.

\section{Cooperative Coverage Extension}

To extend coverage to the UEs located in the service areas of the switched-off BSs and compensate for the SNR loss due to increased BS-UE distances, the nearby active BSs will use cooperative communications and power control. Without loss of generality, we limit the set of cooperating BSs to the six direct neighboring BSs of the switched-off cells, resulting in the BS cooperation modes shown in Fig. 2, Fig. 3 and Fig. 4 for each BS switch-off pattern. Note that switch-off pattern III results in two different cooperative BS geometries and propagation characteristics: mode III (a) (symmetric BS arrangement) occurring with $25 \%$ relative frequency, and mode III (b) (asymmetric BS arrangement) occuring with $75 \%$ relative frequency, as one can tell from the resulting network topology shown in Fig. 4.

When a BS is switched off, it releases its channel resources to its neighboring active BSs. Then the neighboring active BSs use the resources obtained from the switched-off BS to cooperatively serve the UEs inside the switched-off cell. In the cells having an active BS, the UEs are served by the single active BS the same way as during peak-time operation without BS cooperation.

For the UEs inside a switched-off cell, the cooperative coverage extension scheme for both downlink and uplink is specified as follows:

1) Downlink: Since the channel state information is available at the BS side, the optimum cooperative transmission scheme is cooperative beamforming [17]. The resulting SNR at the UE (receiver) is given by:

$$
S N R=\sum_{k=1, m=1}^{K, M} \frac{\left|h_{k}^{(m)}\right|^{2} P_{\mathrm{BS}}}{N_{U E}},
$$

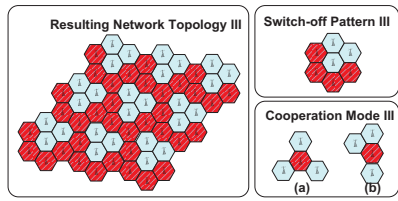

Fig. 4. Switch-off Pattern III: Switching off four out of seven BSs 
where $P_{B S}$ is the total transmit power of all the cooperating BSs, $N_{U E}$ is the noise power at the UE, and $K$ is the number of cooperating BSs.

2) Uplink: For uplink, given that the channel state information is available at the BS side, Maximal Ratio Combining (MRC) [18], [19] is the optimal choice for cooperating BSs. The resulting SNR at the BS (receiver) side is

$$
S N R=\sum_{k=1, m=1}^{K, M} \frac{P_{\mathrm{UE}}\left|h_{k}^{(m)}\right|^{2}}{N_{k}^{(m)}} .
$$

where $P_{\mathrm{UE}}$ is the transmit power of the UE, and $N_{k}^{(m)}$ is the noise power received at the $m$-th antenna of the $k$-th BS. For simplicity, we further assume that $N_{k}^{(m)}$ are identical for all $k$ and $m$, i.e. $N_{k}^{(m)}=N_{B S}$, then

$$
S N R=\frac{P_{\mathrm{UE}}}{N_{B S}} \sum_{k=1, m=1}^{K, M}\left|h_{k}^{(m)}\right|^{2} .
$$

\section{Outage Probability Analysis}

In this paper, we use outage probability as the metric of the users' QoS. For a certain given SNR threshold $\gamma_{0}$, an outage occurs when the instantaneous received SNR is lower than this threshold. To guarantee the QoS for all UEs inside the switched-off cell, a spatially averaged QoS metric is not sufficient. Since a UE can be anywhere within the cell, we will focus on the QoS of the UE situated at the worst transmission/reception location within the switched-off cell.

\section{A. Worst-Case Location}

The first step is to determine the worst-case location within the switched-off cell, given the number and locations of the cooperating active BSs. Fitting their geological locations into a two-dimensional coordinate system, the BS and UE locations are assigned a pair of coordinates. Specifically, we assign coordinates to the cooperating BSs and the UE according to

$$
B S_{1}=\left(x_{1}, y_{1}\right), \cdots, B S_{K}=\left(x_{K}, y_{K}\right) ; U E=(x, y) .
$$

Then, the distance between the UE and $B S_{k}$ can be written as $d_{k}=\left[\left(x-x_{k}\right)^{2}+\left(y-y_{k}\right)^{2}\right]^{\frac{1}{2}}$.

Given the cooperative coverage extension scheme introduced in Section II, the resulting SNR is proportional to the sum $\sum_{k=1, m=1}^{K, M}\left|h_{k}^{(m)}\right|^{2}$. To determine the worst-case location, we will only take into account the effect of path loss by averaging out the fading component:

$$
E\left[\sum_{k=1, m=1}^{K, M}\left|h_{k}^{(m)}\right|^{2}\right]=M \sum_{k=1}^{K} \frac{G}{d_{k}^{\alpha}},
$$

where $K$ is the number of cooperating BSs and $M$ is the number of antennas at each BS.

Consequently, determining the worst-case location within the switched-off hexagonal area is equivalent to solving the following constrained minimization problem:

$$
\begin{array}{rl}
\min _{x, y} & f(x, y)=\sum_{k=1}^{K} \frac{1}{\left[\left(x-x_{k}\right)^{2}+\left(y-y_{k}\right)^{2}\right]^{\frac{\alpha}{2}}} \\
\text { s. t. } & -\sqrt{3} R \leqslant \sqrt{3} x+y \leqslant \sqrt{3} R ; \\
- & \sqrt{3} R \leqslant \sqrt{3} x-y \leqslant \sqrt{3} R ; \\
- & \frac{\sqrt{3}}{2} R \leqslant y \leqslant \frac{\sqrt{3}}{2} R .
\end{array}
$$

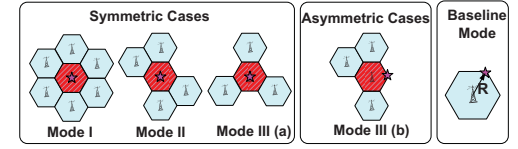

Fig. 5. Worst-case UE locations

For the four different cooperation modes introduced earlier, the solution of the worst-case UE location with $\alpha \in[3,4]$ is given in Fig. 5.

\section{B. Outage Probability with Cooperation}

In this section, we analyze the outage probability of the UEs inside the switched-off cell. Due to the similarity of the SNR expressions for the downlink and uplink transmission scenarios given by (2) and (4), we use the following unified formula in our analysis:

$$
S N R=\sum_{k=1, m=1}^{K, M} \frac{P}{N}\left|h_{k}^{(m)}\right|^{2},
$$

where $P$ and $N$ denote the transmit power and the receiver noise variance, respectively.

1) Symmetric BS Arrangement: For the symmetric cases shown in Fig. 5 (cooperation modes I, II and III (a)), the worstcase location is in the center of the switched-off cell, and the BS-UE distances are the same for all cooperating active BSs. Such a symmetric geometry leads to identical channel gain variances $\sigma_{k}^{2}=\frac{G}{(\sqrt{3} R)^{\alpha}}$, for $k=1, \cdots, K$. Therefore, (7) can be written as

$$
S N R=\frac{P}{N} \frac{\sigma_{k}^{2}}{2} \sum_{k=1, m=1}^{K, M} \frac{2}{\sigma_{k}^{2}}\left|h_{k}^{(m)}\right|^{2} .
$$

Since $W \triangleq \sum_{k=1, m=1}^{K, M} \frac{2}{\sigma_{k}^{2}}\left|h_{k}^{(m)}\right|^{2}$ is a central chi-square random variable with $2 M K$ degrees of freedom, the probability $\operatorname{Pr}[W \leqslant w]$ (i.e. the CDF of $W$ ) is given by

$$
\operatorname{Pr}[W \leqslant w]=\frac{\gamma\left(M K, \frac{w}{2}\right)}{(M K-1) !}
$$

where $\gamma(s, x)=\int_{0}^{x} t^{s-1} e^{-t} d t$ is the lower incomplete Gamma function. Accordingly, the outage probability can be written as

$$
P_{\text {out }}=\operatorname{Pr}\left[S N R \leqslant \gamma_{0}\right]=\frac{\gamma\left(M K, \frac{\gamma_{0} N(\sqrt{3} R)^{\alpha}}{P G}\right)}{(M K-1) !} .
$$

2) Asymmetric BS Arrangement: For the asymmetric case shown in Fig. 5 (cooperation mode III (b)), the distances between the worst-case UE location and the three cooperating BSs are $d_{1}=\sqrt{7} R, d_{2}=2 R$, and $d_{3}=2 R$, respectively. In this case, we rewrite (7) as

$$
S N R=\frac{\sigma_{1}^{2} P}{2 N} \sum_{m=1}^{M} \frac{2}{\sigma_{1}^{2}}\left|h_{1}^{(m)}\right|^{2}+\frac{\sigma_{2}^{2} P}{2 N} \sum_{k=2, m=1}^{K=3, M} \frac{2}{\sigma_{k}^{2}}\left|h_{k}^{(m)}\right|^{2},
$$

where $\sigma_{1}^{2}=\frac{G}{(\sqrt{7} R)^{\alpha}}$, and $\sigma_{2}^{2}=\sigma_{3}^{2}=\frac{G}{(2 R)^{\alpha}}$. Let $Z_{1} \triangleq$ $\sum_{m=1}^{M} \frac{2}{\sigma_{1}^{2}}\left|h_{1}^{(m)}\right|^{2}$ and $Z_{2} \triangleq \sum_{k=2, m=1}^{K=3, M} \frac{2}{\sigma_{k}^{2}}\left|h_{k}^{(m)}\right|^{2}$, which are two central chi-square random variables with $2 M$ and $4 M$ degrees of freedom, respectively. The distribution of the $S N R$ is approximated by that of a scaled chi-square random variable $Z$ with $s$ degrees of freedom in such a way that the variance 
of the $S N R$ in (11) is equal to the variance of $Z$ [20]. Toward this end, we have the following equation to solve for $s$ :

$$
\frac{P^{2}}{N^{2}} \frac{\sigma_{1}^{4}}{2} M+\frac{P^{2}}{N^{2}} \sigma_{2}^{4} M=\frac{1}{s} \frac{P^{2}}{N^{2}}\left(\sigma_{1}^{2} M+2 \sigma_{2}^{2} M\right)^{2},
$$

which yields

$$
s=2 M \frac{\left(\sigma_{1}^{2}+2 \sigma_{2}^{2}\right)^{2}}{\sigma_{1}^{4}+2 \sigma_{2}^{4}}=2 M \frac{\left(7^{-\alpha / 2}+2^{1-\alpha}\right)^{2}}{7^{-\alpha}+2^{1-2 \alpha}} .
$$

Following [20], $Z=\frac{s \cdot S N R}{\frac{P}{N}\left(\sigma_{1}^{2} M+2 \sigma_{2}^{2} M\right)}$ is approximately distributed as a chi-square random variable with $s$ degrees of freedom, so the outage probability can be approximated as

$P_{\text {out }}=\operatorname{Pr}\left[Z \leqslant \frac{\gamma_{0} \cdot s \cdot N}{P M\left(\sigma_{1}^{2}+2 \sigma_{2}^{2}\right)}\right] \approx \frac{\gamma\left(\frac{s}{2}, \frac{\gamma_{0} N R^{\alpha}\left(7^{-\alpha / 2}+2^{1-\alpha}\right)}{P G\left(7^{-\alpha}+2^{1-2 \alpha}\right)}\right)}{\Gamma(s / 2)}$

where $\Gamma(x)=\int_{0}^{\infty} t^{x-1} e^{-t} d t$ is the Gamma function.

3) QoS Requirements: When switching off some of the BSs, it is reasonable to expect that the minimum QoS experienced by the UEs does not worsen compared to the situation where all BSs are active (i.e. peak-hour operation). Therefore, we require that the outage probability of the UE at the worst-case location in the service area of a switched-off BS be less than or equal to the outage probability of a UE at the worst-case location of a cell during peak-hour operation. Since a UE in an active cell is served by a single BS, its worst-case location is at the cell edges, as shown in Fig. 5 labeled as "Baseline mode". The corresponding $S N R$ expression is a special case of (7) with $K=1$ :

$$
S N R=\frac{P}{N} \sum_{m=1}^{M}\left|h_{0}^{(m)}\right|^{2}=\frac{P}{N} \frac{\sigma_{0}^{2}}{2} \sum_{m=1}^{M} \frac{2}{\sigma_{0}^{2}}\left|h_{0}^{(m)}\right|^{2},
$$

where $N$ is the noise power, $P$ is the total transmit power of $\mathrm{BS}$ (donwlink) or UE (uplink), and $h_{0}^{(m)}$ is the channel gain between the UE and the $m$-th antenna of the BS with variance $\sigma_{0}^{2}=\frac{G}{R^{\alpha}}$.

Let $V \triangleq \sum_{m=1}^{M} \frac{2}{\sigma_{0}^{2}}\left|h_{0}^{(m)}\right|^{2}$, which is a central chi-square random variable with $2 M$ degrees of freedom. Thus, the baseline outage probability can be found as

$$
P_{\text {out }}=\operatorname{Pr}\left[V \leqslant \frac{2 \gamma_{0} N}{P \sigma_{0}^{2}}\right]=\frac{\gamma\left(M, \frac{\gamma_{0} N R^{\alpha}}{P G}\right)}{(M-1) !} .
$$

\section{ACHIEVED Power SAVING}

In this section, we quantify the energy saving achieved by the above described energy-efficient network operation scheme. The total power consumption $P_{\text {total }}$ of a BS is divided into two main parts:

- The idle power consumption, represented by $P_{\text {idle, }}$, which does not depend on the network traffic load. This power is consumed even if an active BS is idle (there is no transmission or reception).

- The transmit power consumption, represented by $P_{R F}$, which depends on the network traffic load. This part mainly includes the consumption of the radio frequency (RF) output components.

We will assume that the additional baseband signal processing power consumed due to cooperation among active BSs is negligible compared to the power consumption of conventional (non-cooperative) active BS transmission/reception.
Define $\eta$ to be the ratio of $P_{R F}$ to $P_{\text {total }}$. We also define $\lambda$ as the ratio of the total transmit power required for cooperative transmission to the transmit power required in conventional (non-cooperative) operation to maintain the same minimum QoS level.

During energy-efficient operation, $\beta \times 100 \%$ of $\mathrm{BSs}$ are switched off, resulting in $\beta P_{\text {total }}$ power saving. However, the traffic load from the switched-off BSs is transferred to the remaining active BSs. This means that the total transmit power, and thus $P_{R F}$, will increase by the factor $\lambda$ during energyefficient operation to achieve the required QoS level, reducing the net power saving in the network. Therefore, the percentage of power-saving $\xi$ during energy-efficient operation can be calculated as

$$
\begin{aligned}
\xi & =1-\frac{\text { Total Consumption in Energy-Efficient Operation }}{\text { Total Consumption in Conventional Operation }} \\
& =\frac{\beta P_{\text {total }}-\beta \lambda P_{R F}}{P_{\text {total }}} \\
& =\beta[1-\eta \lambda] .
\end{aligned}
$$

\section{NUMERICAL RESULTS}

In this section, we present some numerical results showing the performance and the energy saving potential of such an energy-efficient cellular network employing the proposed BS switching strategy and cooperative coverage extension scheme.

To characterize the outage probability, we define $\phi=$ $\frac{P}{\gamma_{0} N R^{\alpha} / G}$, where the denominator $\gamma_{0} N R^{\alpha} / G$ is predefined for a given network and fixed in our simulation. The quantity $\phi$ represents a given SNR level normalized by the network- and deployment-dependent system parameters. Thus, the ratio of two $\phi$ values (difference in $d B$ ) is indicative of how much extra transmit power is needed to be able to move from one SNR level to the other.

Fig. 6 and Fig. 7 show the outage probability curves based on (10) (14) and (16) as a function of $\phi$ for the BS switch-off patterns and cooperation modes shown in Fig. 5 with pathloss exponent $\alpha=3.5$ and $\alpha=4$, respectively. The BSs have $M=4$ transmit antennas. The curve labeled as "Baseline QoS Requirement" corresponds to the case when all BSs are active and there is no BS cooperation (i.e. peak-hour operation). From these two figures, one can see that for typical outage probability values (e.g. $10^{-3}-10^{-5}$ ), the QoS level experienced by the switched-off cells can be guaranteed without significant BS transmit power increase due to the performance improvement gained from BS cooperation. In fact, for BS cooperation modes I, II, and III (a), power saving can be observed in both Fig. 6 and Fig. 7. Another observation is that for the asymmetric BS cooperation mode III (b), the approximation in (14) matches the simulation results well, which demonstrates the effectiveness of the approximation described in Section III-B2.

Since $\lambda$ is the ratio of the $\phi$ values corresponding to the "Baseline QoS Requirement" curve and the curve for the desired BS cooperation mode, we can easily find the $\lambda$ values for a given outage probability based on Fig. 6 and Fig. 7. The following table shows the obtained $\lambda$ values (in linear scale) for four different combinations of the path-loss exponent and outage probability. 


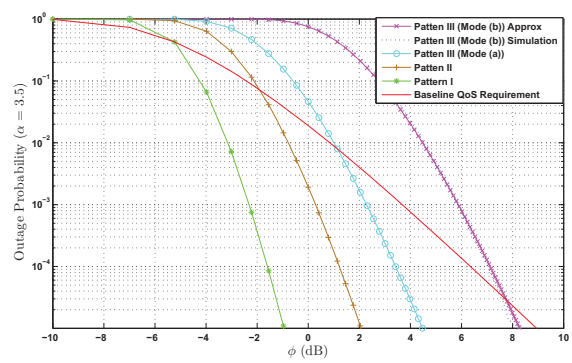

Fig. 6. Outage probability of the switch-off patterns with $\alpha=3.5$

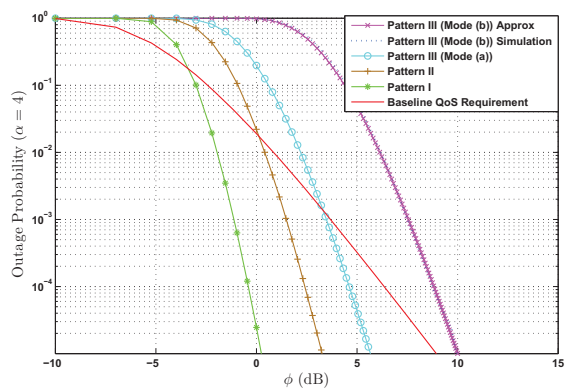

Fig. 7. Outage probability of the switch-off patterns with $\alpha=4$

\begin{tabular}{|c|c|c|c|c|}
\hline$\left(\alpha, P_{\text {out }}\right)$ & $\left(3.5,10^{-3}\right)$ & $\left(3.5,10^{-4}\right)$ & $\left(4,10^{-3}\right)$ & $\left(4,10^{-4}\right)$ \\
\hline I & $\lambda=0.25$ & $\lambda=0.16$ & $\lambda=0.33$ & $\lambda=0.21$ \\
\hline II & $\lambda=0.50$ & $\lambda=0.31$ & $\lambda=0.60$ & $\lambda=0.41$ \\
\hline III(a) $25 \%$ & $\lambda=0.72$ & $\lambda=0.50$ & $\lambda=0.95$ & $\lambda=0.67$ \\
\hline III(b) $75 \%$ & $\lambda=1.66$ & $\lambda=1.17$ & $\lambda=2.46$ & $\lambda=1.75$ \\
\hline III combined & $\lambda=1.43$ & $\lambda=1.11$ & $\lambda=2.08$ & $\lambda=1.48$ \\
\hline
\end{tabular}

Since switch-off pattern III leads to two different cooperation modes III (a) and III (b) occurring with $25 \%$ and $75 \%$ relative frequencies, the $\lambda$ value for the "Mode III combined" case is a weighted sum of the values for modes III (a) and (b), representing the overall $\lambda$ value for BS switch-off pattern III.

The power saving ability of this network, as defined in (17), was evaluated with parameters $\eta=10 \%, \beta_{I}=1 / 3, \beta_{I I}=$ $1 / 2$, and $\beta_{I I I}=4 / 7$. The achieved power saving percentages corresponding to different BS switch-off patterns, path loss exponents and outage probabilities are presented in Fig. 8.

From Fig. 8, we can see that the proposed switch-off patterns and the corresponding BS cooperation modes can result in significant power savings: by operating in energy-efficient mode, the network can save as much as $50 \%$ of its total consumed power depending on the network deployment scenario and the desired QoS level. We can also observe that when $\alpha=3.5$, switching-off more BSs always results in more power saving. However, as $\alpha$ increases (the signal strength attenuates faster with increasing distance), this is not the case any more. As

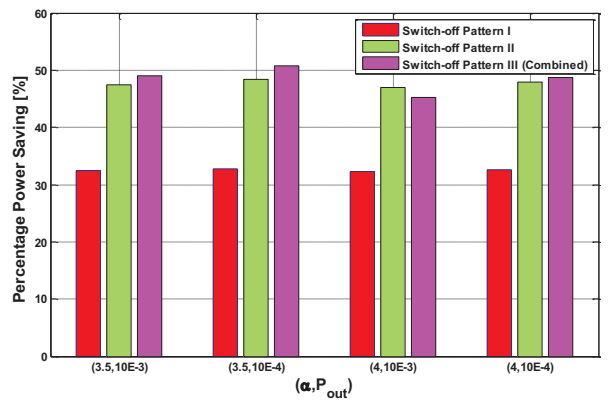

shown in Fig. 8, when $\alpha=4$, aggressively switching off more BSs will not always result in additional power saving since the extra required transmit power to maintain the QoS level can offset the power saving gained by switching off more BSs.

\section{CONCLUSiON}

We proposed three scalable BS switch-off patterns to reduce the power consumption of cellular networks during offpeak hours and used BS cooperation to efficiently extend the network coverage to the service areas of the switchedoff cells. We guaranteed the QoS in those switched-off cells by focusing on the worst-case transmission/reception locations instead of calculating spatial averages. We derived closed form expressions for the outage probability for the considered BS switch-off patterns and cooperation modes and evaluated the achievable power saving. A potential of up to $50 \%$ power saving was observed in our numerical results.

\section{REFERENCES}

[1] G. Fettweis and E. Zimmermann, "ICT energy consumption-trends and challenges", Proc. of the 11th International Symposium on Wireless Personal Multimedia Communications (WPMC), 2008.

[2] "Core 5 - Green radio: programme objectives and overview," Presentation from Mobile VCE. Available at http://www.mobilevce.com/dloads-publ/ mtg284Item_1503.ppt

[3] M. Marsan et al., "Optimal energy savings in cellular access networks", IEEE International Conference on Communications (ICC), June 2009.

[4] E. Oh et al., "Towards dynamic energy-efficient operation of cellular network infrastructure", IEEE Communications Magazine, pp. 56-61, June 2011.

[5] L. Chiaravigilo et al., "Energy-aware UMTS access networks", International Symposium on Wireless Personal Multimedia Communications (WPMC), September 2008.

[6] E. Oh and B. Krishnamachari, "Energy savings through dynamic base station switching in cellular wireless access networks", IEEE Global Communications Conference (Globecom), December 2010.

[7] S. Zhou et al., "Green mobile access network with dynamic base station energy saving", ACM International Conference on Mobile Computing and Networking (MobiCom), September 2009.

[8] A. Fehske, F. Richter and G. Fettweis, "Energy efficiency improvement through micro sites in cellular mobile radio networks", IEEE Global Communications Conference (Globecom), December 2009.

[9] K. Son, E. Oh and B. Krishnamachari, "Energy-aware hierarchical cell configuration: from deployment to operation", IEEE International Conference on Computer Communications (INFOCOM), April 2011.

[10] M. Marsan and M. Meo, "Energy efficient management of two cellular access networks", ACM SIGMETRICS, June 2009.

[11] K. J. Ray Liu, Ahmed Sadek, Weifeng Su, and Andres Kwasinski, Cooperative Communications and Networking, Cambridge University Press, Jan., 2009.

[12] A.K. Sadek, W. Yu, and K.J.R. Liu, "On the Energy Efficiency of Cooperative Communications in Wireless Sensor Networks", ACM Trans. on Sensor Networks, vol 6, no 1, Dec 2009.

[13] A.K. Sadek, Z. Han, and K.J.R. Liu, "Distributed Relay-Assignment Protocols for Coverage Extension in Cooperative Wireless Networks", IEEE Trans. on Mobile Computing, vol 9, no 4, pp.505-515, April 2010.

[14] D. Cao et al., "Energy saving performance comparison of coordinated multi-point transmission and wireless relaying", IEEE Global Communications Conference (Globecom), December 2010.

[15] R. M. Karp, "Reducibility among combinatorial problems", Complexity of Computer Computations, pp. 85-103, 1972.

[16] M. R. Garey and D. S. Johnson, Computers and Intractability: A Guide to the Theory of NP-Completeness, $1^{s} t$ ed., W. H. Freeman Jan., 1979

[17] H. Ochiai, P. Mitran, H. V. Poor and V. Tarokh, "Collaborative beamforming for distributed wireless ad hoc sensor networks", IEEE Trans. Signal Process., vol. 53, no, 11, pp. 4110-4124, Nov. 2005.

[18] D. G. Brennan, "On the maximum signal-to-noise ratio realizable from several noisy signals", Proc. IRE, vol. 43, p. 1530, Oct. 1955.

[19] A. J. Goldsmith, Wireless Communication, $1^{\text {st }}$ ed., New York: Cambridge University, 2005.

[20] F. E. Satterthwaite, "Synthesis of variance", Psychometrika, vol. 6, no. 5, pp. 309-316, Oct. 1941.

Fig. 8. The achieved power saving 